\title{
Estudo da vascularização folicular e do corpo lúteo de éguas cíclicas tratadas com extrato de pituitária equina utilizando ultrassom Doppler colorido
}

\author{
[Study of follicular and corpus luteum vascularization in mares treated with Equine \\ Pituitary Extract using ultrasound color Doppler] \\ M.C.R. Medeiros ${ }^{1}$, A. Farinasso ${ }^{2}$, I.H.A.V. Nery ${ }^{1}$, H.M. Souza ${ }^{1}$, J.L.A.Vasconcelos ${ }^{1}$, \\ K.L.G. Trindade ${ }^{1}$, A.M. Batista ${ }^{1}$, H.C. Manso Filho ${ }^{1}$, G.F. Carneiro ${ }^{1}$, A. Wischral ${ }^{1}$ \\ ${ }^{1}$ Universidade Federal Rural de Pernambuco - UFRPE - Recife, PE \\ ${ }^{2}$ Laboratório Unicórnio - Brasília, DF
}

\begin{abstract}
RESUMO
Informações sobre a vascularização da parede folicular e do corpo lúteo equino, associadas à superovulação, são escassas. Com o objetivo de avaliar o efeito superovulatório do extrato de pituitária equina (EPE) no fluxo sanguíneo folicular e luteal, foram utilizadas seis éguas Puro Sangue Árabe, em dois ciclos estrais (controle e tratamento). As éguas foram monitoradas diariamente por ultrassonografia modo B, até que os folículos atingissem diâmetro de $23 \mathrm{~mm}$ (desvio). No ciclo tratamento, as éguas receberam $8 \mathrm{mg}$ de EPE, uma vez ao dia, por via IM, até que dois ou mais folículos atingissem o diâmetro entre 32 e $35 \mathrm{~mm}$. A ovulação foi induzida com acetato de deslorelina, quando os folículos atingiram, no mínimo, $35 \mathrm{~mm}$. No momento do desvio folicular, da indução da ovulação e do último exame préovulatório, foi utilizada a ultrassonografia modo B para medir o diâmetro dos folículos e, no oitavo dia pós-ovulação, para a área do corpo lúteo (CL). Utilizou-se também ultrassonografia com Doppler colorido para avaliar a perfusão sanguínea da parede folicular e do parênquima luteal. No ciclo controle, foi realizado o mesmo procedimento, exceto pelo uso do EPE. Os dados foram submetidos à análise de variância, com nível de significância de 5\%. Não foi observado efeito do EPE sobre o número de ovulações, o diâmetro dos folículos, a vascularização da parede folicular e a concentração sérica de estrógeno. Os animais, tratados ou não, apresentaram CLs funcionais, não havendo diferença na área do parênquima ou da vascularização luteal, nem na concentração sérica de progesterona, no oitavo dia após a ovulação. Foi observado que o EPE proporcionou um maior número de folículos subordinados no momento da indução da ovulação do folículo dominante $(\mathrm{P} \leq 0,05)$. Embora esses folículos não tenham chegado a ovular, concluiu-se que o EPE atuou no crescimento de folículos, que podem ser utilizados em outras biotécnicas, como a transferência de oócitos, com maior aproveitamento da reserva folicular de ovários equinos.
\end{abstract}

Palavras-chave: equino, folículo ovariano, fluxo sanguíneo, superovulação

\begin{abstract}
Knowledge about follicle and corpus luteum vascularization associated with superovulation in mares is scarce. Aiming to evaluate the effect of equine pituitary extract (EPE) on superovulation, the experiment was conducted using six mares Purebred Arabian in two estrous cycles (control and treatment). The mares were synchronized, and monitored daily by ultrasound B mode until the follicles reached diameter $\leq 23 \mathrm{~mm}$ (deviation). In the treatment cycle, from the deviation, mares received $8 \mathrm{mg}$ of EPE, once a day, intramuscularly, until two or more follicles reached a diameter between 32 and $35 \mathrm{~mm}$. Ovulation was induced with deslorelin acetate when follicles reached at least $35 \mathrm{~mm}$. At the time of follicular deviation, induction of ovulation and final preovulatory exam, it was used B-mode ultrasound to measure the diameter of follicles and on the eighth day after ovulation to measure the area of the corpus luteum (CL); color Doppler was also used to assess blood perfusion of the follicle wall and luteal parenchyma. In the
\end{abstract}

Recebido em 8 de novembro de 2016

Aceito em 3 de dezembro de 2016

E-mail: mariavet_mcrm@hotmail.com 
control cycle was performed the same procedure except for the use of EPE. Data were subjected to analysis of variance, with 5\% significance level. There was no effect of EPE on ovulation number, diameter of follicles, vascularity of the follicular wall and serum estrogen concentration. The animals treated or not, showed functional CLs, with no difference in parenchymal area or luteal vascularization, or in serum progesterone concentration on the eighth day after ovulation. It was observed that the EPE provided a greater number of subordinate follicles at the time of induction of ovulation of the dominant follicle. Although these follicles have failed to ovulate, it was concluded that EPE influenced the follicles growth, and it can be used in other biotechnologies, with greater utilization of equine ovarian follicular reserve.

Keywords: equine, ovarian follicle, blood flow, superovulation

\section{INTRODUÇÃO}

O ciclo reprodutivo da égua se caracteriza por uma fase folicular seguida de fase luteal, decorrente, na maioria das vezes, de uma única ovulação. Na ocorrência da emergência de uma onda folicular ovulatória, os folículos se desenvolvem em uma fase de crescimento comum até o início do desvio, quando o folículo dominante continua a crescer e os folículos subordinados começam a regredir. O desvio começa quando o futuro folículo dominante apresenta cerca de $22,5 \mathrm{~mm}$, sendo a capacidade para a dominância semelhante entre os quatro maiores folículos, no início do desvio (Ginther et al., 2004).

Para aumentar a taxa de produção embrionária equina, vários protocolos hormonais já foram utilizados, mas sem grande sucesso. Todavia, o extrato de pituitária equina (EPE) tem sido utilizado com considerável sucesso, principalmente em nível experimental (Pimentel e Carneiro, 2008).

A superovulação de éguas com EPE, em diferentes protocolos, tem aumentado significativamente o número de ovulações e, com isso, a obtenção de mais de um embrião por ciclo (Dippert et al., 1994; Scoggin et al., 2002; Rocha Filho, 2005; Squires e McCue, 2007). Constatouse que o tratamento com dose diária baixa foi eficiente para induzir múltiplas ovulações em éguas cíclicas (Farinasso et al., 2005). O EPE também tem sido utilizado para induzir a ovulação em animais em fase de transição reprodutiva (Barros, 2015).

O acompanhamento da dinâmica folicular de éguas vem sendo realizado com ultrassonografia modo B. Mais recentemente, a ultrassonografia com Doppler colorido vem sendo usada, pois é uma técnica não invasiva que possibilita avaliar a fisiologia do fluxo sanguíneo do trato reprodutivo, incluindo a vascularização ovariana na égua (Ferreira e Meira, 2011; Ayres e Mingoti, 2012).

A vascularização da parede folicular ovariana em éguas tem apresentado maior fluxo sanguíneo no futuro folículo dominante, até mesmo dois dias antecedentes à ocorrência do desvio (Acosta et al., 2004). Witt et al. (2012), ao estudarem tratamento superovulatório com hormônios gonadrotóficos em éguas, verificaram que houve um aumento da perfusão ovariana. Por outro lado, no folículo pré-ovulatório, há uma redução na vascularização da parede, cerca de dois dias antes de a ovulação ocorrer (Gastal et al., 2007).

A eficiência reprodutiva na espécie equina está aumentando cada vez mais após o incremento da reprodução assistida (Pimentel e Carneiro, 2008) e, nos últimos anos, estudos demonstraram que a superovulação na espécie equina tem sido uma alternativa para buscar uma maior taxa de recuperação embrionária. Mas, considerando que os resultados ainda não são satisfatórios, estudos sobre os efeitos do tratamento superovulatório na dinâmica e vascularização folicular precisam ser realizados. Para isso, a ultrassonografia utilizada como ferramenta de monitoramento da foliculogênese tem sido de relevância fundamental, principalmente em centrais de transferência de embrião. No entanto, o monitoramento com ultrassonografia com Doppler colorido ainda é pouco utilizado, principalmente em associação à superovulação. Com isso, o presente estudo propôs avaliar o efeito do EPE, na dinâmica folicular, na perfusão sanguínea folicular e luteal, bem como na esteroidogênese de éguas cíclicas. 


\section{MATERIAL E MÉTODOS}

Foram utilizadas seis éguas da raça Puro Sangue Árabe, com idade entre nove e 20 anos, em regime de criação semiextensivo, alimentadas com pastagem de capim nativo e feno $6 \mathrm{~kg} /$ dia/animal, além de ração de manutenção (2,5kg/dia/animal), sal mineral e água ad libitum. As éguas pertenciam ao Departamento de Zootecnia da Universidade Federal Rural de Pernambuco, localizada em Dois Irmãos, Recife, PE (8 1' 3" S e 34 56' 58" W).

Todas as éguas deste estudo inicialmente tiveram o ciclo estral sincronizado utilizando-se uma dose luteolítica de prostaglandina $\mathrm{F}_{2 \alpha}$ ( $1 \mathrm{mg}$, IM, Dinoprost Trometamina, Lutalyse ${ }^{\circledR}$, Zoetis, Brasil).

O experimento foi realizado no período entre fevereiro e maio de 2015, em duas etapas ou ciclos estrais, sendo um ciclo controle e outro tratamento. Aleatoriamente, as éguas que foram avaliadas inicialmente no ciclo controle e no ciclo posterior foram submetidas ao tratamento com extrato de pituitária equina (EPE). Já aquelas éguas que foram inicialmente submetidas ao tratamento superovulatório, após seu término tiveram um ciclo de descanso, que não foi aproveitado para análise e, posteriormente à ovulação deste ciclo, foi iniciada a avaliação do ciclo controle. Este procedimento considerou que não há efeito residual do EPE sobre os ovários no ciclo seguinte ao tratamento (Farias, 2013).

No ciclo tratamento, foi administrado EPE, de uma partida extraída de éguas que estavam em anestro estacional, um produto experimental que foi cedido pelo Laboratório Unicórnio, Brasília, DF, sob responsabilidade técnica do Dr. Adalberto Farinasso.

O tratamento com EPE (8mg, uma vez ao dia, IM) foi iniciado no momento do desvio de um ou mais folículos em crescimento (entre 20 e $23 \mathrm{~mm}$ ) e a aplicação diária foi interrompida quando a égua apresentou um ou mais folículos com diâmetro entre 32 a $35 \mathrm{~mm}$ ou após ter recebido o total de seis doses.
Para indução da ovulação, utilizou-se o acetato de deslorelina $\left(0,75 \mu \mathrm{g}\right.$, por via IM, Sincrorrelin ${ }^{\circledR}$ - Ourofino, Brasil) em éguas com folículos entre de 35-40mm e edema uterino superior a grau 2.

As éguas avaliadas no ciclo controle receberam os mesmos procedimentos, exceto pela administração do EPE.

Tanto no ciclo controle quanto no tratado com EPE, após a aplicação de $\mathrm{PGF}_{2 \alpha}$, as éguas foram monitoradas diariamente por ultrassonografia modo $\mathrm{B}$ até o desvio do diâmetro folicular. A partir do desvio, os folículos foram avaliados também pelo modo Doppler colorido para se avaliar a perfusão sanguínea folicular até a ovulação ocorrer.

Para realização dos exames ultrassonográficos, foi utilizado um aparelho de ultrassonografia equipado com Doppler colorido (Mindray - M5 Vet), sendo os folículos e corpos lúteos (CLs) examinados de maneira lenta e contínua, de forma a permitir a visualização dos sinais coloridos correspondentes aos vasos sanguíneos (perfusão) em toda a parede folicular e parênquima luteal (Fig. 1). Todos os exames foram gravados para análise posterior. Durante a análise, os folículos foram subjetivamente divididos em quatro quadrantes, e o percentual de parede contendo pontos coloridos foi somado e considerado como o total de perfusão da parede folicular. Os CLs foram avaliados da mesma forma; o parênquima foi também dividido em quadrantes e considerou-se o percentual de área com pontos coloridos como o percentual de vascularização do CL (Ginther e Utt, 2004).

A inseminação artificial, com sêmen fresco foi realizada, no máximo, após 24 horas da indução da ovulação com deslorelina e, no oitavo dia após a ovulação, procedeu-se à coleta do embrião. Para lavagem uterina, utilizou-se soro ringer lactato, em torno de 3 a 4 litros, realizando-se o procedimento pelo sistema aberto. No mesmo dia da coleta de embrião, a égua recebeu uma dose de prostaglandina $\mathrm{F}_{2 \mathrm{a}}$ para lise de corpo lúteo. 


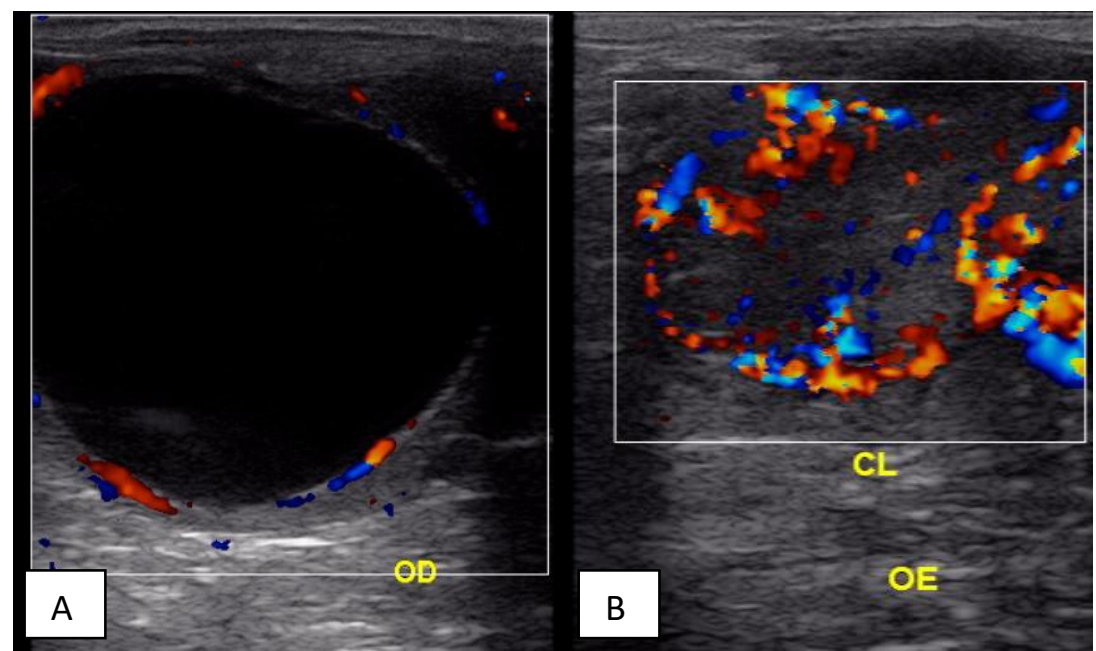

Figura 1. Imagens ultrassonográficas com Doppler colorido de folículo (A) e de corpo lúteo (B), para análise de perfusão em éguas.

Os embriões foram classificados de acordo com o Manual da Sociedade Internacional de Transferência de Embriões (Robertson e Nelson, 1998), lavados em tampão fosfato salino (PBS) suplementado com $1 \mathrm{mg} / \mathrm{mL}$ de polivinilpirrolidona (PVP) e fixados em paraformaldeído 4\% em PBS, durante uma hora, na temperatura ambiente. Após duas lavagens em PBS/PVP, os embriões foram permeabilizados em $0,5 \%$ de Triton $\mathrm{X}-100$ e $0,1 \%$ de citrato de sódio em PBS, durante uma hora na temperatura ambiente. Em seguida, os embriões foram lavados três vezes em PBS/PVP e incubados em gotas contendo Hoescht $33342(10 \mathrm{mg} / \mathrm{mL})$ e iodeto de propídeo $(125 \mathrm{mg} / \mathrm{mL})$ por 15 minutos no escuro.

Em seguida, os embriões foram lavados três vezes em PBS/PVP, montados em lâminas e cobertos com lamínulas e, então, examinados em microscópio de epifluorescência utilizando-se os filtros $350 \mathrm{~nm}$ e $488 \mathrm{~nm}$. Cada embrião foi analisado para determinação da viabilidade. Os blastômeros que fluoresceram em azul foram considerados viáveis (com membrana plasmática íntegra), enquanto aqueles que fluoresceram em vermelho ou rosa foram considerados inviáveis. Entretanto, embriões com menos de $50 \%$ de células vermelhas ou rosas foram considerados viáveis.

Amostras de sangue foram coletadas, para dosagem de estrógeno sérico, quando pelo menos um folículo ultrapassou o desvio de diâmetro e imediatamente antes da indução da ovulação com deslorelina (folículo com diâmetro entre de 35$40 \mathrm{~mm}$ ). Para dosagem da concentração de progesterona sérica, foram coletadas amostras de sangue no oitavo dia após a ovulação.

A dosagens foram realizadas por ensaio imunoenzimático (ELISA) utilizando-se testes comerciais (Estradiol Sym e Progesterona Sym, Symbiosis Diagnóstica Ltda., Brasil). A sensibilidade do teste foi de $6,5 \mathrm{pg} / \mathrm{mL}$ e $0,157 \mathrm{ng} / \mathrm{mL}$, e o coeficiente de variação intraensaio foi de $14,9 \%$ e $11,8 \%$, respectivamente, para estrógeno e progesterona.

As variáveis concentração de estrógeno e progesterona, diâmetros foliculares, número de folículos mensuráveis, percentuais de vascularização da parede dos folículos ovulatórios e do parênquima luteal e área luteal foram avaliadas por análise de variância (ANOVA), e a diferença entre os grupos (controle e tratamento) testada pelo teste de Tukey. Para análise de correlação entre variáveis, foi utilizado o teste de Spearman. Valores foram considerados significativos quando $\mathrm{P} \leq 0,05$.

\section{RESULTADOS E DISCUSSÃO}

Este estudo avaliou a dinâmica do diâmetro e da vascularização folicular e a concentração de estrógeno circulante após tratamento com EPE em éguas. Não foram observadas diferenças significativas $(\mathrm{P}>0,05)$ no diâmetro e na vascularização folicular entre os grupos tratado e não tratado com EPE, nos três momentos 
avaliados: dia do desvio, dia da indução da ovulação com acetato de deslorelina e préovulação (Tab. 1).

Esses dados corroboram os resultados de Farias (2013), que não observou diferença significativa ao comparar o tamanho médio de folículos préovulatórios de éguas das raças Crioula e Quarto de Milha tratadas ou não com EPE. Contudo, Gimenes (2010) verificou que éguas tratadas com EPE, recebendo $8 \mathrm{mg}$ duas vezes ao dia, a partir do momento em que os folículos atingissem 20-23mm de diâmetro, apresentaram maior número de ovulações quando comparadas ao grupo controle. Porém, esse autor utilizou o dobro da dose utilizada no presente estudo.

Tabela 1. Média e desvio-padrão dos níveis de estrógeno $\left(\mathrm{E}_{2}\right)$ circulante, diâmetro $(\varnothing)$ e percentual $(\%)$ de vascularização do folículo dominante e número $(\mathrm{N})$ total de folículos e ovulações em éguas tratadas com EPE

\begin{tabular}{lcc}
\hline & Controle & EPE \\
\hline $\mathrm{N}$ & 6 & 6 \\
$\mathrm{E}_{2}$ no desvio (pg/mL) & $126,1 \pm 46,3$ & $146,8 \pm 86,5$ \\
$\mathrm{E}_{2}$ na indução da ovulação (pg/mL) & $152,4 \pm 147,9$ & $142,6 \pm 112,3$ \\
& & \\
$\varnothing$ no desvio (mm) & $22,7 \pm 1,0 \mathrm{~A}$ & $22,5 \pm 1,2 \mathrm{~A}$ \\
$\varnothing$ na indução da ovulação (mm) & $37,7 \pm 2,6 \mathrm{~B}$ & $38,2 \pm 7,3 \mathrm{~B}$ \\
$\varnothing$ na pré-ovulação (mm) & $42,3 \pm 5,6 \mathrm{~B}$ & $42,6 \pm 7,8 \mathrm{~B}$ \\
$\varnothing$ máximo do folículo dominante $(\mathrm{mm})$ & $42,1 \pm 5,1$ & $42,3 \pm 9,1$ \\
$\varnothing$ máximo do folículo subordinado $(\mathrm{mm})$ & $22,7 \pm 8,0$ & $23,4 \pm 6,9$ \\
& & \\
\% vascularização no desvio & $48,7 \pm 8,8 \mathrm{a}$ & $50,8 \pm 18,7 \mathrm{a}$ \\
\% vascularização na indução da ovulação & $47,5 \pm 10,1$ & $57,3 \pm 17,4$ \\
\% vascularização na pré-ovulação & $48,3 \pm 14,2$ & $53,0 \pm 12,2$ \\
& & \\
$\mathrm{~N}$ de folículos no desvio (20-23mm) & $1,8 \pm 0,9 \mathrm{a}$ & $3,2 \pm 1,3 \mathrm{a}$ \\
$\mathrm{N}$ de folículos na indução da ovulação & $2,8 \pm 1,2 \mathrm{a}$ & $4,6 \pm 1,0 \mathrm{~b}$ \\
$\mathrm{~N}$ de ovulações & $1,0 \pm 0,0 \mathrm{a}$ & $1,3 \pm 0,5 \mathrm{a}$ \\
\hline
\end{tabular}

ab - Letras diferentes na linha representam diferença significativa $(\mathrm{P}<0,05)$. $\mathrm{AB}$ - letras diferentes na coluna, para uma mesma variável entre tempos, representam diferença significativa $(\mathrm{P}<0,05)$.

Witt et al. (2012) verificaram que éguas cíclicas tratadas com eFSH não demonstraram diferença significativa no fluxo sanguíneo ovariano em relação ao grupo controle, decorrente de haver um ou dois folículos em crescimento durante todo o período de avaliação. No entanto, houve um maior fluxo sanguíneo ovariano em éguas com mais de dois folículos, nos ciclos tratados com eFSH, porém este estudo foi realizado avaliando-se o fluxo da artéria ovariana e não da parede folicular.

Não foi observado efeito do EPE na concentração de estrógeno circulante (Tab. 1), no entanto foi detectada uma fraca, mas significativa, correlação dos níveis de estrógeno circulante com a vascularização folicular no momento do desvio $\left(\mathrm{P}=0,05 ; \mathrm{r}^{2}=0,38\right)$ e no dia da indução da ovulação $\left(P=0,05 ; r^{2}=0,33\right)$, independentemente do tratamento realizado. $\mathrm{O}$ efeito vasodilatador do estradiol já é conhecido e deve ser responsável pelo aumento do fluxo sanguíneo dos folículos, conforme já constatado no fluxo sanguíneo ovariano em éguas (Witt et al., 2012) e em vacas (Honnens et al., 2009). É importante ressaltar que os estudos realizados para avaliar o efeito da superovulação na vascularização se detiveram em avaliar o fluxo das artérias ovarianas, por meio dos índices de pulsatividade e resistividade, mas não na microvascularização folicular, como realizado nesta pesquisa.

Gastal et al. (1999a) detectaram que o nível de estradiol aumenta no início do desvio do futuro folículo dominante. Acosta et al.(2004) notaram fluxo sanguíneo maior no futuro folículo dominante cerca de um a dois dias antes do desvio em diâmetro. No entanto, faltam estudos mais aprofundados sobre a relação entre o nível de estrógeno, no desvio folicular, e a vascularização do futuro folículo dominante. 
Gastal et al. (2007) observaram queda no nível de estradiol nos dois dias antecedentes à ovulação, porém, com a aproximação da ovulação, a vascularização da parede folicular aumenta. Por outro lado, essa vascularização reduz a partir de seis horas antes da ovulação (Ginther et al., 2007). Na presente pesquisa, essa redução não foi constatada, provavelmente devido à frequência dos exames (a cada 24 horas).

As variações da onda folicular estão associadas às alterações nos níveis circulantes de FSH e LH e à capacidade dos folículos responderem às gonadotrofinas (Donadeu e Pedersen, 2008). O desvio folicular se dá a partir do momento em que os dois maiores folículos alteram a taxa de crescimento diário (Gastal et al., 1999b). Nesse estudo, o número médio de folículos em crescimento no momento do desvio folicular foi comparado entre o grupo controle e o grupo tratado com EPE, não apresentando diferença significativa $(\mathrm{P}>0,05)$.

Já o tratamento com EPE resultou em maior número de folículos em crescimento, no momento da indução com deslorelina, do que os animais não tratados $(\mathrm{P}=0,016)$. Resultados semelhantes foram encontrados por Blanco et al. (2009) ao realizarem tratamento com EPE (25mg), com ou sem associação ao hCG (2500U.I.), verificando-se uma contribuição significativa para o aumento do número de folículos disponíveis.

Embora esse maior número de folículos recrutados não tenha ovulado, no grupo tratado com EPE (Tab. 1), pode-se sugerir a utilização de outra biotécnica, como aspiração folicular, para aproveitamento desses oócitos.

A fraca resposta de ovulação corrobora Rocha Filho (2005), que não verificou diferença no número médio de ovulações entre o tratamento com dose reduzida de EPE (4mg/dia) e seu controle. No presente estudo, o tratamento foi realizado com $8 \mathrm{mg} /$ dia de EPE, que pode também ser considerada uma baixa dose, comparada com 12,5 a 50mg em diferentes frequências (Scoggin et al., 2002) e 14mg/dia (Boninet al., 2010; Farias, 2013), que resultaram em efeito positivo no número de ovulações. Outro aspecto a ser considerado é que a partida do hormônio utilizada neste estudo foi obtida de animais em anestro estacional, teoricamente apresentando reduzida quantidade de FSH e LH no produto final em relação ao esperado para as formulações obtidas de éguas em fase cíclica.

Das éguas submetidas a tratamento de superovulação, quatro foram inseminadas artificialmente com sêmen fresco e tiveram embriões coletados no oitavo dia, resultando em três embriões (um de cada égua) que apresentavam boa qualidade morfológica e integridade celular. Das éguas controle, três foram inseminadas e submetidas à coleta de embrião; foram obtidos dois embriões, com qualidade similar aos das éguas tratadas (Fig. 2).

Todas as éguas se encontravam em período de ciclos normais durante o experimento, apresentando concentrações séricas de progesterona elevadas (média superior a $11 \mathrm{ng} / \mathrm{mL}$ ) no oitavo dia.

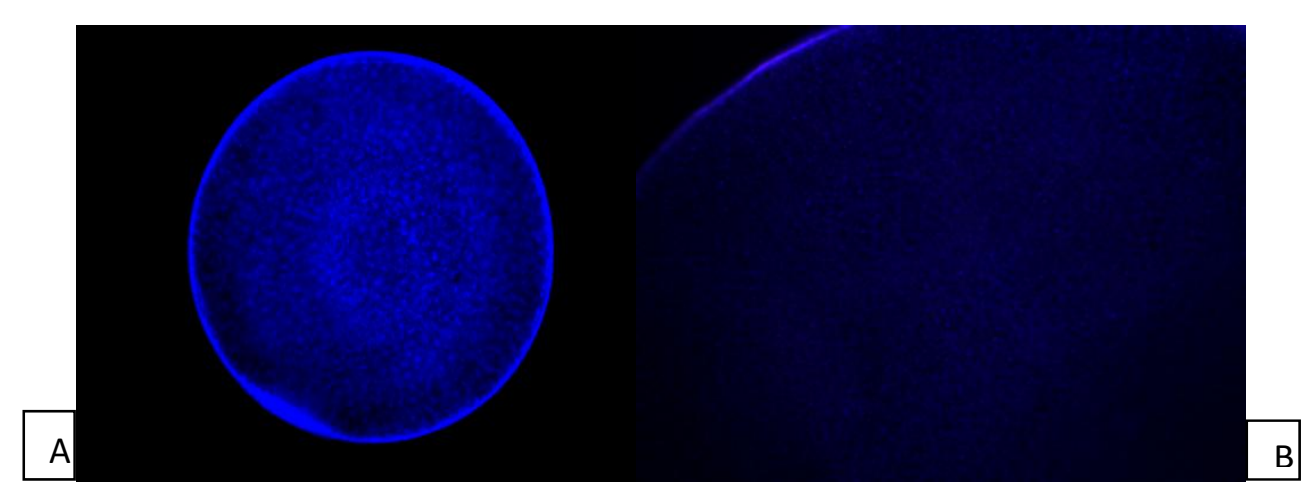

Figura 2. Imagem de embriões coletados de égua controle (A) e tratada com EPE (B). A coloração azul indica células íntegras. 
Não foi constatado efeito do tratamento do EPE sobre a função luteal $(\mathrm{P}>0,05)$ (Tab. 2). Os níveis séricos de progesterona foram semelhantes tanto no grupo controle quanto no grupo tratado com EPE, também verificado em resultados encontrados por Ginther et al. (2007), em que a progesterona atingiu o máximo de concentração no dia oito $(12,8 \mathrm{ng} / \mathrm{mL})$.

No presente estudo, não foi encontrada correlação entre vascularização, área luteal e nível sérico de progesterona, corroborando Gomes (2008) e Maia (2015), que estudaram o efeito da indução da ovulação com acetato de deslorelina em éguas, e não encontraram nenhuma correlação significativa entre essas variáveis.

A perfusão sanguínea luteal foi avaliada oito dias após a ovulação, apresentando alto percentual de vascularização. Alonso (2013) verificou que o máximo de vascularização ocorreu no oitavo dia após a ovulação, independentemente de o animal ser tratado com hCG ou controle. Já Ginther et al. (2007) encontraram resultados diferentes, observando que o fluxo sanguíneo luteal máximo ocorreu no dia 10 após ovulação. Todavia, Bollwein et al. (2002), ao avaliarem o ciclo estral de éguas com idade acima de sete anos, constataram que a vascularização luteal cresceu até o dia sete após a ovulação, quando começou a declinar.

Tabela 2. Média e desvio-padrão dos níveis de progesterona $\left(\mathrm{P}_{4}\right)$ circulante, área luteal total $\left(\mathrm{cm}^{2}\right)$ e percentual $(\%)$ de vascularização do corpo lúteo, oito dias após a ovulação, em éguas tratadas com EPE

\begin{tabular}{lcc}
\hline & Controle & EPE \\
\hline $\mathrm{N}$ & 6 & 6 \\
$\mathrm{P}_{4}(\mathrm{ng} / \mathrm{mL})$ & $11,97 \pm 2,51$ & $11,64 \pm 3,08$ \\
Área total $\left(\mathrm{cm}^{2}\right)$ & $5,24 \pm 0,86$ & $5,91 \pm 2,0$ \\
$\%$ vascularização & $82,66 \pm 8,1$ & $83,16 \pm 4,4$ \\
\hline
\end{tabular}

\section{CONCLUSÃO}

O extrato de pituitária equina - partida obtida de éguas durante anestro estacional - não foi eficiente para provocar pelo menos a dupla ovulação em todas as éguas tratadas, não afetou a dinâmica folicular com relação ao crescimento (diâmetro) e à vascularização da parede, tampouco influenciou os níveis plasmáticos de estrógeno e progesterona em éguas cíclicas da raça Puro Sangue Árabe. Por outro lado, o EPE proporcionou um recrutamento maior de folículos presentes no dia da indução da ovulação.

\section{AGRADECIMENTOS}

Ao Dr. Adalberto Farinasso, pela concessão do EPE; ao Prof. Helio Manso Cordeiro Filho, pela concessão dos animais; e à Fundação de Amparo à Ciência e Tecnologia do Estado de Pernambuco (Facepe), pela bolsa de estudos.

\section{REFERÊNCIAS}

ACOSTA, T.J.; GASTAL, E.L.; GASTAL, M.O. et al. Differential blood flow changes between the future dominant and subordinate follicles precede diameter changes during follicle selection in mares. Biol. Reprod., v.71, p.502-507, 2004.

ALONSO, M.A. Efeito da aplicação de hCG em diferentes dias do ciclo estral sobre a concentração sérica de progesterona e fluxo sanguíneo uterino e ovariano em éguas. 2013. 188f. Tese (Doutorado em Reprodução Animal) - Faculdade de Medicina Veterinária e Zootecnia, Universidade de São Paulo, São Paulo, SP.

AYRES, H.; MINGOTI, G.Z. Angiogênese, vascularização e uso do ultrassom Doppler colorido na avaliação de estruturas ovarianas. Rev. Bras. Reprod. Anim., v.36, p.174-180, 2012.

BARROS, M.B.S. Indução de ovulação com acetato de deslorelina e extrato de pituitária equina em éguas cíclicas em períodos transicionais. 2015. 56f. Dissertação (Mestrado em Ciência Veterinária) Universidade Federal Rural de Pernambuco, Recife, PE.

BLANCO, I.D.; DEVITO, L.G.; FERREIRA, H.N. et al. Aspiration of equine oocytes from immature follicles after treatment with equine pituitary extract (EPE) alone or in combination with hCG. Anim. Reprod. Sci., v.114, p.203-209, 2009.

BOLLWEIN, H.; MAYER, R.; WEBER, F.; STOLLA, R. Luteal blood flow during the estrous cycle in mares. Theriogenology, v.57, p.2043-2051, 2002.

BONIN, B.F.; DELL'AQUA JUNIOR, J.A.; FIORATTI, E.G.; ALVARENGA, M.A. Efeito do tratamento com extrato de pituitária equina na resposta ovariana e eficiência reprodutiva de éguas idosas em programa de transferência de embriões. Vet. Zootec., v.17, p.94-103, 2010. 
DIPPERT, K.D.; JASKO, D.J.; SEIDEL JR., G.E.; SQUIRES, E.L. Fertilization rates in superovulated and spontaneously ovulating mares. Theriogenology, v.41, p.1411-1423, 1994.

DONADEU, F.X.; PEDERSEN, H.G. Follicle development in mares. Reprod. Domest. Anim., v.43, p.224-231, 2008.

FARIAS, D.K. Protocolo superovulatório com extrato de pituitária equina (EPE) em éguas da raça Crioula e Quarto de Milha. 2013. 45f. Dissertação (Mestrado em Ciência Animal) - Centro de Ciências Agroveterinárias da Universidade do Estado de Santa Catarina, Lages, SC.

FARINASSO, A.; BRANQUINHO, J.A.; RUMPF, R.; ALVARENGA, M.A. Utilização de baixas doses de extrato de pituitária equina (EPE) para indução de ovulações múltiplas em éguas. Acta Sci. Vet., v.33, p.135-138, 2005

FERREIRA， J.C.; MEIRA， C. Aplicação da ultrassonografia colorida Doppler em programas de transferência de embriões equinos. Cienc. Rural, v.41, p.1063-1069, 2011.

GASTAL, E.L.; GASTAL, M.O.; DONANDEU, F.X. et al. Temporal relationships among LH, estradiol, and follicle vascularization preceding the first compared with later ovulations during the year in mares. Anim. Reprod. Sci., v.102, p.314-321, 2007.

GASTAL, E.L.; GASTAL, M.O.; GINTHER, O.J. Experimental assumption of dominant by a smaller follicle and associated hormonal changes in mares. Biol. Reprod., v.61, p.724-730, 1999a.

GASTAL, E.L.; GASTAL, M.O.; WILTBANK, M.C.; GINTHER, O.J. Follicle deviation and intrafollicular and systemic estradiol concentrations in mares. Biol. Reprod., v.61, p.31-39, 1999b.

GIMENES, A.M. Resposta Ovariana em éguas tratadas com baixa dose de extrato de pituitária equina. 2010. 74f. Dissertação (Mestrado em Reprodução Animal) - Faculdade de Medicina Veterinária e Zootecnia, Universidade Estadual Paulista "Júlio de Mesquita Filho", Botucatu, SP.

GINTHER, O.J.; GASTAL, E.L.; GASTAL, M.O. et al. Dose-response study of intrafollicular injection of insulin-like growth factor-I on follicular fluid factors and follicle dominance in mares. Biol. Reprod., v.70, p.1063-1069, 2004.

GINTHER, O.J.; GASTAL, E.L.; GASTAL, M.O. et al. Luteal blood flow and progesterone production in mares. Anim. Reprod. Sci., v.99, p.213-220, 2007.
GINTHER, O.J.; UTT, M.D. Doppler ultrasound in equine reproduction: principles, techniques, and potential. J. Equine Vet. Sci., v.24, p.516-526, 2004.

GOMES, T.J.S. Equine corpus luteum vascular evaluation by power Doppler ultrasound. 2008. 66f. Dissertação (Mestrado em Medicina Veterinária) Faculdade de Medicina Veterinária, Universidade Técnica de Lisboa, Lisboa.

HONNENS, A.; WEISSER, S.; WELTER, H. et al. Relationships between ovarian blood flow and ovarian response to eCG-treatment of dairy cows. Anim. Reprod. Sci., v.113, p.1-10, 2009.

MAIA, V.N. Avaliação da vascularização $e$ da angiogênese de corpos lúteos de éguas com ovulação natural e induzida por acetato de deslorelina. 2015. 67f. Tese (Doutorado em Ciência Animal Tropical) Universidade Federal Rural de Pernambuco, Recife, PE.

PIMENTEL, C.A.; CARNEIRO, G.F. Biotécnicas aplicadas à reprodução de equinos. In: GONÇALVES, P.B.D.; FIGUEIREDO, J.R.; FREITAS, V.J.F. (Eds.). Biotécnicas aplicadas à reprodução animal. São Paulo: Roca, 2008. p.145-159.

ROBERTSON, I.; NELSON, R.E. Certificação e identificação de embriões. In: STRINGFELLOW, D.A.; SEIDEL, S.M. (Eds.). Manual da sociedade internacional de transferência de embriões. Savory: IETS, 1998. p.109-140.

ROCHA FILHO, A.N. Efeito do tratamento com baixa dose de extrato de pituitária ou FSH purificado equino no crescimento folicular, taxa de ovulação $e$ recuperação embrionária em éguas. 2005. 64f. Dissertação (Mestrado em Reprodução Animal) Faculdade de Medicina Veterinária e Zootecnia, Universidade Estadual Paulista "Júlio de Mesquita Filho", Botucatu, SP.

SCOGGIN, C.F.; MEIRA, C.; MCCUE, P.M. et al. Strategies to improve the ovarian response to equine pituitary extract in ciclic mares. Theriogenology, v.58, p.151-164, 2002.

SQUIRES, E.L.; MCCUE, P.M. Superovulation in mares. Anim. Reprod. Sci., v.99, p.1-8, 2007.

WITT, M.C.; BOLLWEIN, H.; PROBST, J. et al. Doppler sonography of the uterine and ovarian arteries during a superovulatory program in horses. Theriogenology, v.77, p.1406-1414, 2012. 